\title{
Channeling of Developing Rat Corticospinal Tract Axons by Myelin- Associated Neurite Growth Inhibitors
}

\author{
M. E. Schwab and L. Schnell \\ Institute for Brain Research, University of Zurich, $\mathrm{CH}-8029$ Zurich, Switzerland
}

\begin{abstract}
CNS myelin contains 2 membrane proteins that are potent inhibitors of neurite growth (NI-35 and NI-250). Because myelin formation starts at different times in different regions and tracts of the CNS, this inhibitory property of myelin could serve boundary and guidance functions for late-growing fiber tracts. In the rat, the corticospinal tract (CST) grows into and down the spinal cord during the first 10 postnatal days, in close proximity to the sensory tracts fasciculus cuneatus and gracilis. Immunofluorescence for myelin constituents showed that, in the rostral half of the spinal cord, the myelinating tissue of these ascending tracts surrounds the growing, myelin-free CST in a channellike fashion. Elimination of oligodendrocytes by $x$-irradiation of the newborn rats, or application of antibody IN-1, which neutralizes the inhibitory substrate property of CNS myelin, resulted in significant anatomical aberration of CST flbers. In particular, the tract was larger in cross-section, and aberrant CST fibers and fascicles intermixed with the neighboring sensory ascending tracts. These results assign an important channeling and "guard-rail" function to the oligodendrocyte-associated neurite growth inhibitors for the developing CST in the rat spinal cord.
\end{abstract}

Substrate interactions play a crucial role for cell migration, fiber outgrowth, and tract formation in the developing PNS and CNS (Sanes, 1983; Dodd and Jessell, 1988; Jessell, 1988). In the CNS, growing axons often follow glial end-feet regions, astrocyte processes, or other axons. Such guidance phenomena seem to depend on a number of favorable substrate molecules on these cells and structures. In addition to substrates and factors enhancing neurite growth, negative interactions and molecules with repulsive or inhibitory properties were recently described. Growth arrest and growth cone retraction have been observed when neurites of different neuronal types interacted in vitro (Kapfhammer et al., 1986; Kapfhammer and Raper, 1987; Raper and Kapfhammer, 1990). Long-lasting arrest of neurite growth occurs when neuronal growth cones contact oligodendrocytes or CNS myelin in culture (Schwab and Caroni, 1988). In the

\footnotetext{
Received Oct. 30, 1989; revised Oct. 22, 1990; accepted Oct. 24, 1990.

We thank our colleagues Drs. D. Cadelli, J. Kapfhammer, and T. Savio for critically reading the manuscript, Dr. P. Streit for the anti-HRP hybridoma cells, Mrs. S. Kaufmann for secretarial help, and Mr. R. Schoeb for photographic assistance. The work was supported by Swiss National Science Foundation Grant 3.065.87 and by grants from Regeneron Pharmaceuticals (Tarrytown, NY), the American Paralysis Association, The Swiss Multiple Sclerosis Society, and the Dr. E. Slack-Gyr Foundation (Zurich).

Correspondence should be addressed to Martin E. Schwab, Brain Research Institute, University of Zurich, August-Forel-Strausse 1, CH-8029 Zurich, Switzerland.

Copyright (C) 1991 Society for Neuroscience $0270-6474 / 91 / 110709-13 \$ 03.00 / 0$
}

developing visual system, membranes of the posterior part of the developing chicken or goldfish optic tectum were avoided by the axons of the temporal, but not of the nasal retina, results that suggest a role of repulsive constituents in the formation of the retinotopic map (Walter et al., 1987; Vielmetter and Stürmer, 1989). In vivo, transitory structures, often of glial nature, were observed in places suggesting a boundary function for growing axons (Silver et al., 1987; Steindler and Cooper, 1987; Hankin and Silver, 1988). The presence of tenascin $/ \mathrm{Jl}_{220} \mathrm{im}-$ munoreactivity in the developing barrel field of the rat somatosensory cortex in a pattern delineating barrels at an early time (Steindler et al., 1989) may be of particular interest, because purified tenascin is known to be a poor substrate for several cell types in vitro (Chiquet-Ehrismann et al., 1988).

The first biochemically identified constituents with a very putent inhibitory action on growing neurites were 2 proteins found in CNS myelin and oligodendrocyte membranes (Caroni and Schwab, 1988a). These neurite growth inhibitors (NI-35 and NI-250) can abolish the favorable substrate properties of, for example, peripheral nerve membranes. On the other hand, their neutralization by antibodies allowed neurite growth on CNS myelin or oligodendrocytes in culture and led to massive neurite ingrowth into otherwise totally nonpermissive optic nerve explants in vitro (Caroni and Schwab, 1988b; Bandtlow et al., 1990). Recent experiments also showed long-distance regeneration of lesioned corticospinal tract axons in the rat spinal cord in the presence of these antibodies (Schnell and Schwab, 1990) or after suppression of myelin formation (Savio and Schwab, 1990).

Only limited information is available on the developmental expression and possible physiological role of these neurite growth inhibitors, NI-35 and NI-250. In the optic nerve, oligodendrocytes and inhibitory activity appear simultaneously with the first differentiated oligodendrocytes, shortly after the peak number of axons has been reached (Caroni and Schwab, 1989). In the spinal cord and, in fact, in all brain regions, oligodendrocyte differentiation and myelin formation occur in a heterochronous way in the various tracts, layers, and nuclei (Windle et al., 1934; Bensted et al., 1957; Rozeik and Von Keyserlingk, 1987; Schwab and Schnell, 1989). Thus, late-growing fiber tracts can border on tracts and areas already containing differentiated oligodendrocytes and myelin. In the present work, we examined the hypothesis that oligodendrocyte-associated neurite growth inhibitors could channel late-growing CNS tracts by forming boundaries and by exerting a "guard-rail" function. The corticospinal tract (CST) of the rat was chosen as a model system, because it enters the developing spinal cord at birth and forms a solid, well-defined tract containing about 100,000 axons over the first 10 postnatal days (Schreyer and Jones, 1982; Gribnau 
Figure 1. Dorsal funiculi of a P8 spinal cord at the cervical level, double labeled for the myelin marker $\mathrm{O}_{1} / \mathrm{GalC}$ $(a)$, and the CST of the right side $(b)$. $a$, The sensory ascending tracts (st) fasciculus cuneatus $(\mathrm{cu})$ and gracilis $(\mathrm{gr})$ are strongly positive for $\mathrm{O}_{1}$. In contrast, the CST territory is free of myelin. $b$, The CST on the right side is labeled by anterograde transport of RITC. Note the sharp dorsal border to the ascending sensory tracts (arrow). At this age, the CST still contains growing axons (Schreyer and Jones, 1988). Magnification, $156 \times$.
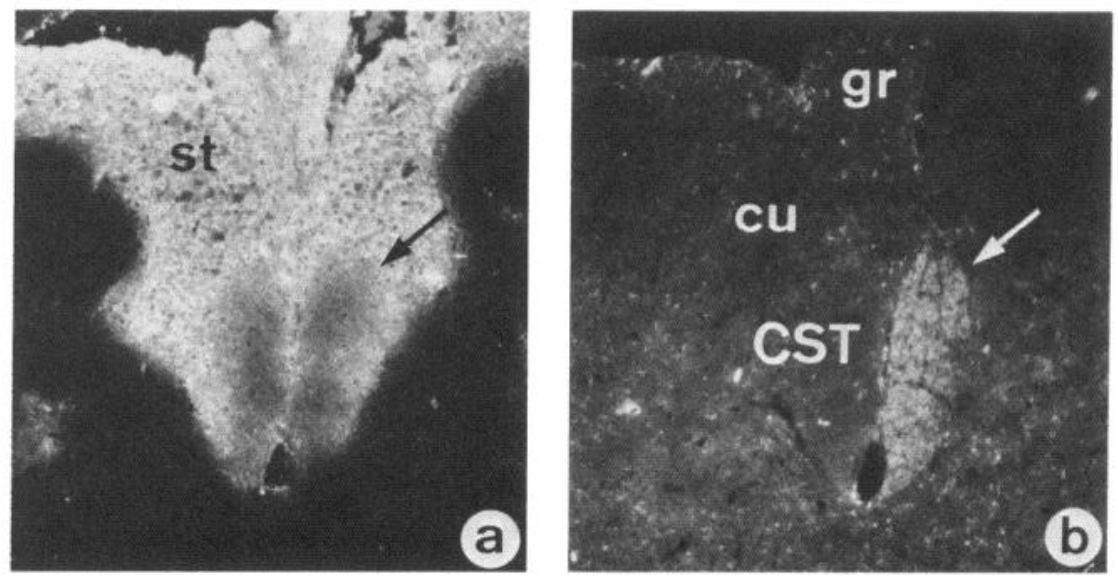

et al., 1986; Schreyer and Jones, 1988). A conspicuous scattering and ectopic growth of CST fibers was observed in spinal cords where oligodendrocyte development and myelin formation were prevented by $\mathrm{x}$-irradiation and in rats after application of a monoclonal antibody neutralizing the neurite growth inhibitors NI-35 and NI-250.

\section{Materials and Methods}

Lewis rats were timed by the day of birth (P0), or, for the early postnatal time course of CST and myelin development, from the day of conception. CST development was studied at $6 \mathrm{hr}, \mathrm{Pl}-4, \mathrm{P} 7, \mathrm{P} 8$, and P9-12, using 3-5 litters (4-6 rats per litter) for each time point and experimental condition.

\section{Experimental treatments}

Controls. No treatment was made before the tracing procedure.

$X$-irradiated animals. Newborn rats (2-8 hr after birth) were anesthetized by hypothermia. The spinal cord or parts of it were irradiated by $X$ rays $(5,500 \mathrm{rad}$ for $5 \mathrm{~min} ; 50 \mathrm{kV} / 14 \mathrm{~mA}$ at a focus distance of 20 $\mathrm{cm} ; 0.25 \mathrm{~mm}$ aluminum filter; Gilmore, 1963a). The rest of the body was protected by a lead shield.

IN-1 antibody rats. IN-1 hybridoma cells were obtained from a fusion after immunization of a mouse with NI-250 (Caroni and Schwab, 1988b). IN-1 antibodies neutralize the inhibitory activity of NI-250, NI-35, and total CNS myelin (Caroni and Schwab, 1988b). Newborn rats (2-8 hr after birth) were anesthetized on ice, and $2 \mu \mathrm{l}$ of an IN-1 cell suspension containing approximately $10^{6}$ cells and a high concentration of antibodies were injected through the skull into the right parietal cortex using a $10-\mu \mathrm{l}$ Hamilton syringe. Tumor-bearing animals selected for examination at ages later than $\mathrm{P} 4$ received cyclosporin $\mathrm{A}(15 \mu \mathrm{g} / \mathrm{gm}$ in olive oil, i.p.) at P3 and P6.

Control-antibody rats. Hybridoma cells secreting antibodies against HRP (not inhibiting HRP enzymatic activity) were generated from the same parent myeloma line (P3U) and were used as controls. Newborn rats were injected as described above.

Presence and size of the well-vascularized hybridoma cell tumors were monitored by dissection and visual inspection of the brains after fixation for all the antibody-treated animals. In selected cases, the tumor sites were inspected histologically, and IN-1 levels were determined by radioimmunoassay (using CNS myelin-coated tubes and ${ }^{125}$ I-anti-mouse $\mathrm{Ig}$ ) in the blood and cerebrospinal fluid (CSF; collected at the time of fixation).

\section{CST visualization by anterograde labeling with wheat germ agglutinin-horseradish peroxidase (WGA-HRP)}

For WGA-HRP tracing, animals were anesthetized on ice $(\mathrm{P} 0-4)$ or by ether (P7-12) and injected with $0.5 \mu \mathrm{l} \mathrm{WGA-HRP(SIGMA;} 5 \%$ in water) superficially into the left frontoparietal cortex. Twenty-four hours later, animals were anesthetized with ether and perfused through the heart with a prerinse of Ringer's solution containing heparin, followed by
$1.25 \%$ glutaraldehyde $/ 1 \%$ formaldehyde in $0.1 \mathrm{M}$ phosphate buffer for $10 \mathrm{~min}$. The whole spinal cords were dissected and postfixed for $1 \mathrm{hr}$ in the same fixative. Twenty-five-micron frozen sections were cut in the transverse plane at various levels, or alternatively, sagittal section series were prepared. Sections were mounted on poly-D-lysine coated objective slides and reacted for HRP activity using tetramethylbenzidine (TMB) as a substrate (Mesulam, 1978). Sections were dehydrated, embedded in Eukitt, and viewed under dark-field and polarization optics in an Olympus Vanox T microscope. In some rats, the CST was labeled by injection of rhodamine isothiocyanate (RITC; SIGMA; $2.5 \%$ in phosphate-buffered saline with $2 \%$ dimethylsulfoxide).

\section{Quantification of CST cross-sectional area}

Dark-field micrographs of spinal cord cross-sections were taken at P10 at cervical level 4 (C4) and thoracic level 5 (T5) for all experimental groups ( $n=9-17$ rats for each group). The CST area was measured by a graphic tablet and the SIGMASCAN program (Jandel Scientific, Sausalito, CA). Significance levels were determined by the Student's $t$ test.

\section{Immunofluorescence}

Myelin formation in normal $\mathrm{P} 0-4$ and $\mathrm{P} 8-10$ animals, in $\mathrm{x}$-irradiated spinal cords, and in IN-1-treated rats was monitored using antibodies $\mathrm{O}_{1}$ [recognizing galactocerebroside $(\mathrm{GalC})$; Sommer and Schachner, 1981], and antibodies against myelin basic protein (MBP; Boehringer Mannheim, Germany). Antibodies against glial fibrillary acidic protein (GFAP; Dakopatts, Denmark) were used as astrocyte marker.

Rats were fixed by perfusion with $4 \%$ formaldehyde in phosphate buffer, and $25-\mu \mathrm{m}$ frozen sections were reacted with the $\mathrm{O}_{1}$ antibody (hybridoma culture supernatant) for $30 \mathrm{~min}$. For MBP and GFAP staining, sections were pretreated for $30 \mathrm{~min}$ by immersion in ice-cold $95 \%$ ethanol $/ 5 \%$ acetic acid. Anti-mouse Ig-fluorescein isothiocyanate (FITC for $\mathrm{O}_{1}$ and MBP; Cappel) or anti-rabbit Ig-FITC (for GFAP) were used as secondary antibodies.

\section{Results}

Myelin formation in the dorsal funiculus and development of the CST in normal rats

In the rat, the dorsal funiculus is formed by 3 major tracts: the sensory ascending tracts fasciculus cuneatus and fasciculus gracilis and the descending CST. These tracts develop and myelinate at very different times; whereas the ascending tracts have stopped growing and start myelination immediately after birth (Matthews and Duncan, 1971; Schwab and Schnell, 1989), the CST just enters the spinal cord at birth and starts myelination at P10-11 (Schreyer and Jones, 1982; Schwab and Schnell, 1989). Growing CST axons thus border on the myelinating sensory tracts (Fig. 1). Because neurite growth inhibitors are expressed by differentiating oligodendrocytes (Caroni and Schwab, 1989), 
we investigated the time course and arrangement of the sensory tract myelin with regard to the ingrowing CST fibers using immunofluorescence for MBP and anterograde labeling of the CST with the highly sensitive marker WGA-HRP. Anterograde tracing of the CST with WGA-HRP was performed and analyzed at $6 \mathrm{hr}$ postnatal and at $\mathrm{P} 1, \mathrm{P} 2, \mathrm{P} 3, \mathrm{P} 4$, and $\mathrm{P} 8-12$ on longitudinal sections of the whole cervical and thoracic spinal cord and on cross-sections taken at the levels $\mathrm{C} 4$ and $\mathrm{T} 5$. The results confirm the descriptions by Schreyer and Jones (1982) and Gribnau et al. (1986; Fig. 2). CST fibers enter the cervical segments of the spinal cord around birth, and early-growing axons reach the lower thoracic level around P3 and the lumbar level around P4-5. Myelin formation starts in the fasciculus cuneatus at birth and follows a rostrocaudal gradient. MBP immunofluorescence at the levels $\mathrm{C} 4$ and $\mathrm{T} 5$ at $6 \mathrm{hr}$ postnatal, at $\mathrm{Pl}$, and at $\mathrm{P} 2$ is shown in Figure 3. The myelination in the fasciculus cuneatus precedes the ingrowth of the CST (Fig. $3 a, d, e$ ). Interestingly, a relatively sharp dorsal boundary of the future CST region is outlined by the myelinating cuneate axons. At P4, myelinating axons are arranged around the growing CST, forming a channellike structure in the cervical and upper thoracic region (Fig. $4 a, b)$. In the lower thoracic and lumbar region, however, myelin formation is much less advanced, and no such "channel" is visible (Fig. $4 c, d$ ). Thus, a difference exists between the rostral half of the spinal cord, where myelinating cuneate axons form a channellike structure before CST axons grow in, and the caudal half of the spinal cord, where no "myelin channel" is observed. Labeling of the CST and analysis of its boundaries led to an interestingly complementary picture: 'the dorsal CST boundary is extremely sharp in the cervical and upper thoracic region (Fig. $4 e, f)$, but CST fascicles arc intcrmixing with the sensory tracts in the lower thoracic and lumbar region (Fig. $4 e, g$ ). It is also noteworthy that the "myelin channel" is thick on the dorsal and dorsolateral aspect of the CST, but sparse and incomplete on the ventrolateral aspect and absent ventrally (Fig. $4 a, b$ ). It is precisely in the latter 2 areas that collaterals innervating the gray matter leave the CST (from P3 on in the cervical cord; Schreyer and Jones, 1982; cf. also Figs. $7 a, 9 a$ ). These findings correlate well with a local inhibitory effect of myelin-associated neurite growth inhibitors on the growing CST axons. This local inhibition would prevent the ingrowth and intermixing of descending CST fibers with ascending sensory tracts, especially in the rostral half of the spinal cord. In order to test this hypothesis, myelin formation was prevented by $\mathrm{x}$-irradiation, and in a second set of experiments, neurite growth inhibitors were specifically neutralized by a monoclonal antibody.

\section{Growth of CST fibers in $x$-irradiated, myelin-free spinal cords}

$\mathrm{X}$-irradiation of the spinal cord in freshly born rats has been shown to almost completely wipe out the oligodendrocytes and to result in myelin-free spinal cords (Gilmore, 1963a,b). Figure 5 illustrates the great reduction of 2 myelin markers, GalC and $\mathrm{MBP}$, in an $\mathrm{X}$-irradiated $\mathrm{P} 10$ spinal cord. In contrast, the radially arranged, GFAP-positive astrocyte processes were still present in the "white matter." Examination of the CST cross-section on the levels $\mathrm{C} 4$ and $\mathrm{T} 5$ of these spinal cords showed a doubling of the tract area (Figs. 6, 7). CST axons also appeared more scattered; in particular, CST fibers and fascicles were now also present in the ascending sensory tracts in the rostral half of the spinal cord (Fig. $7 b-d$ ). Some fibers coursed to the contralateral side of the spinal cord, a feature not seen in the controls (Fig. 7).

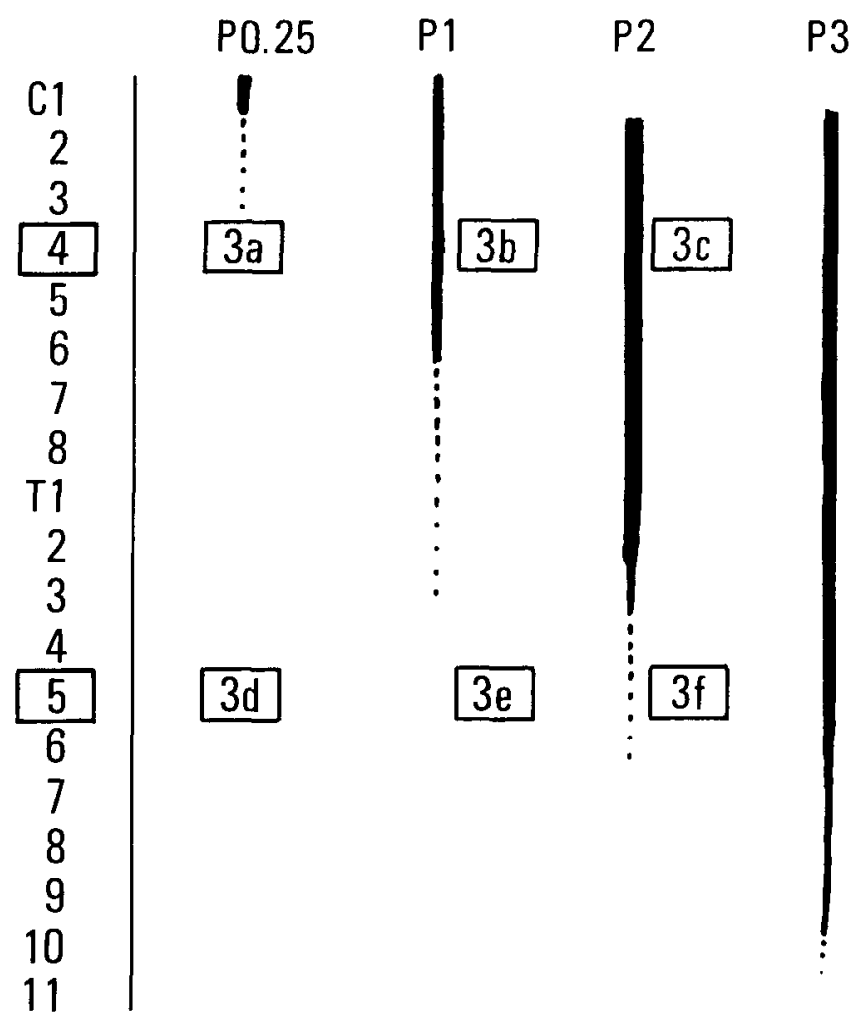

Figure 2. Growth of CST axons into the rat spinal cord at $6 \mathrm{hr}(P 0.25)$ and $P I-3$. Spinal cord levels are indicated as cervical $(C)$ and thoracic (T). Dotted lines indicate axons preceding the bulk of the CST axons by 2-4 segments. Myelin formation at the levels $\mathrm{C} 4$ and T5 is illustrated in Figure 3 ; boxes $3 a-f$ correspond to the individual plates of Figure 3 .

Complete series of sagittal sections comprising lower cervical, thoracic, and upper lumbar levels of P4 and P9-12 control and $\mathrm{x}$-irradiated rats were analyzed for the occurrence of aberrant CST axons within the sensory fascicles of the dorsal funiculus. Sections were chosen at a parasagittal level close to the midline, a region corresponding to the center of the "channel" shown in Figures 1 and 2. As described above, in normal rats, a very sharp boundary was observed between the CST and the ascending tracts in the cervical and upper thoracic region (Fig. $8 a, e)$. In $\mathrm{x}$-irradiated rats, in contrast, CST fibers entering the sensory tracts could be seen at all levels, including the cervical and upper thoracic spinal cord. These fibers descended in the sensory tracts over long distances (Fig. $8 b-d, f$ and thus were most probably true CST axons rather than axon collaterals. At the lower thoracic and lumbar level, the number of CST fibers/ fascicles in the ascending tracts was greatly increased. When the lumbar part of the spinal cord was spared from irradiation, no "damming up" of aberrant CST fibers was seen at the caudal end of the irradiation zone (not shown). Fibers close to the CST and in the sensory tracts seemed to join the main CST again; other aberrant fibers probably ended locally in gray matter within the irradiated segments. As is obvious in transverse (Fig. 7) and sagittal sections (Fig. 8), the amount of CST collaterals in gray matter is also increased in the myelin-free rats at $\mathrm{P} 10$ and P4.

\section{CST development in the presence of $I N-1$ antibodies}

The monoclonal antibody IN-1 neutralizes the neurite growth inhibitors NI-35 and NI-250 as well as the inhibitory activity 

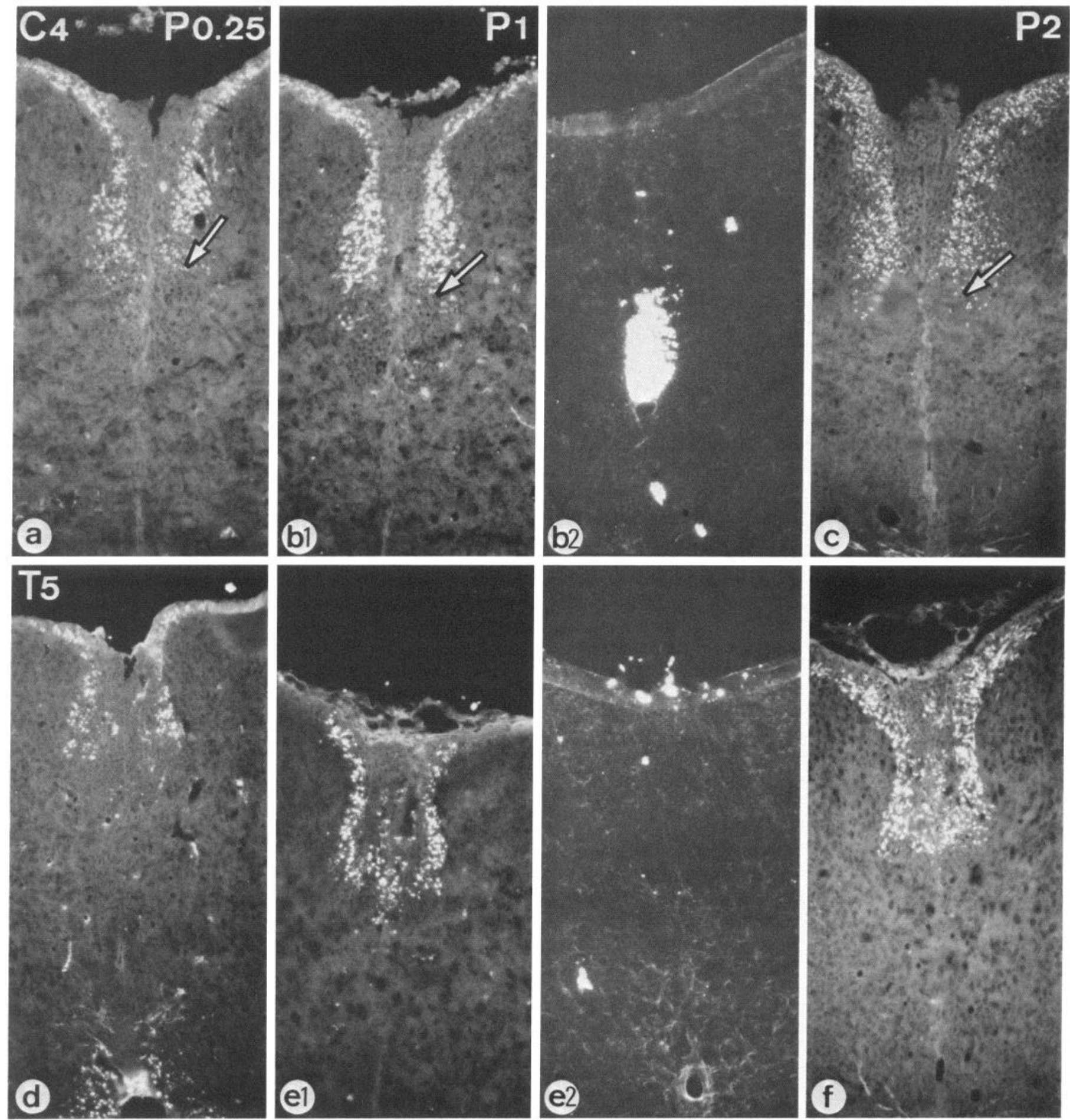

Figure 3. Myelin formation as demonstrated by immunofluorescence for MBP in the dorsal funiculi of the spinal cord at the cervical level $C 4$ $(a-c)$ and the thoracic level $T 5(d-f)$ at $6 \mathrm{hr}(P 0.25), P 1$ and $P 2$. Compare with Figure 2 for the corresponding CST development. At P0.25 (a,d) myelinating fasciculus cuneatus axons outline the dorsal border of the future CST area before CST axons grow in (arrows). A similar picture is seen at P1 at the thoracic level $(e l) . b 2$ and $e 2$ show anterogradely labeled CST axons (WGA-HRP), present at the level C4 (b2) but still absent at T5 (e2). A sharp border is visible between the myelinating cuneate area and the CST area at P2 (c, $f)$. Magnification, $70 \times$.

Figure 4. Myelin formation $(a-d)$ and CST anatomy $(e-g)$ at various spinal levels in P4 $(a-e)$ and P10 $(f, g)$ rats. $a-d$, Immunofluorescence for MBP demonstrates a channellike structure formed by myelinating fasciculus cuneatus axons surrounding the CST ( $a$ and $b$, arrows) at the cervical $(a)$ and upper thoracic $(b)$ level. In contrast, myelin is more sparse and does not form a channel at the lower thoracic $(c)$ and lumbar $(d)$ level. Note also the thin and incomplete myelin border on the ventrolateral and ventral aspect of the CST, where collaterals grow out into the gray matter at this time $(a, b), e$, Montage of spinal cord sagittal section showing the WGA-HRP-labeled CST. Whereas the dorsal boundary of the tract is sharp at the cervical and upper thoracic level, fascicles of CST axons intermix with ascending sensory tracts at the lower thoracic and lumbar level (arrows). $f$ and $g$, Higher magnification of the CST in a P10 rat showing the sharp boundary between sensory tracts (st) and CST at the cervical 
The Journal of Neuroscience, March 1991, $11(3) 713$
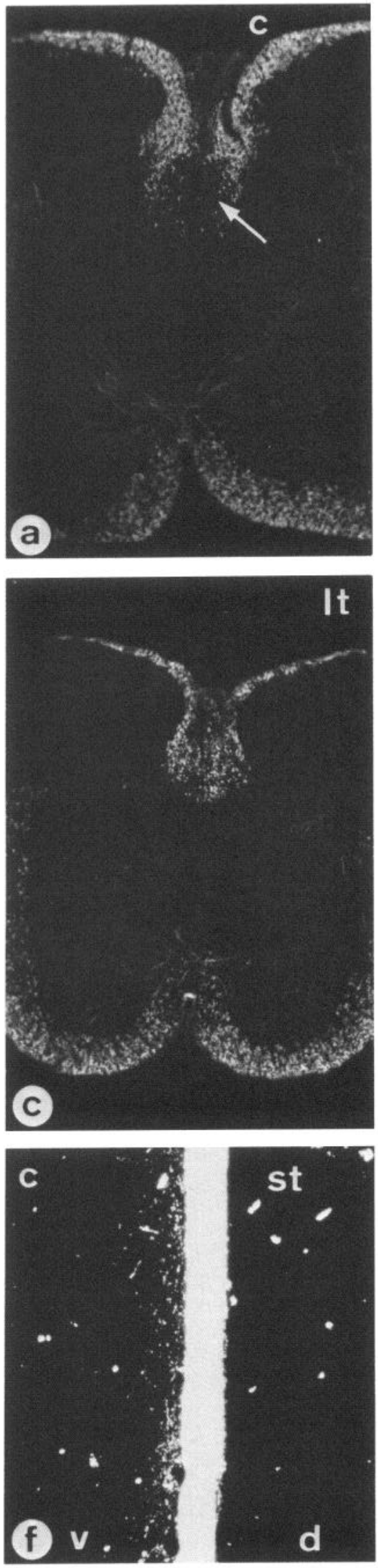
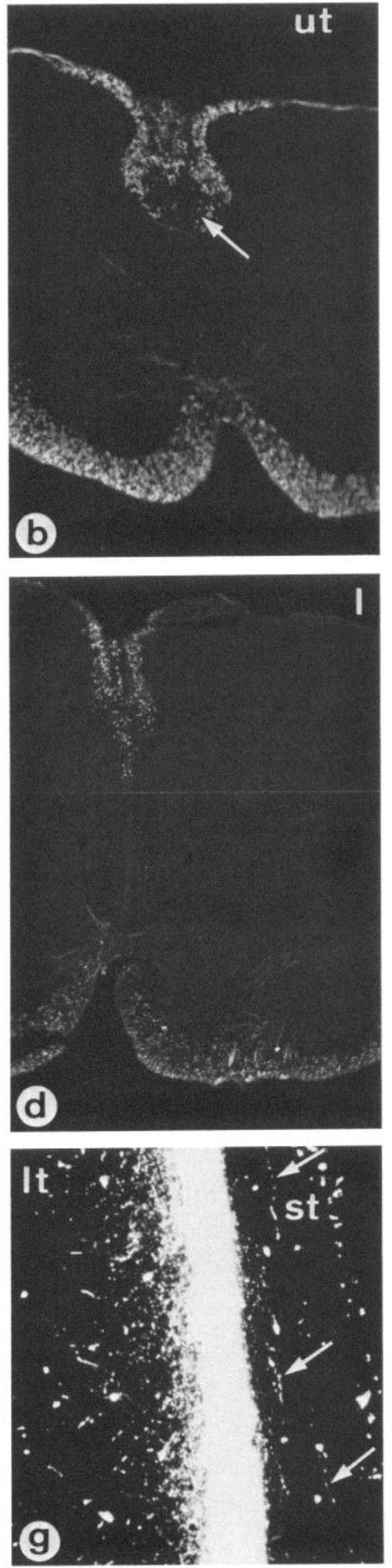

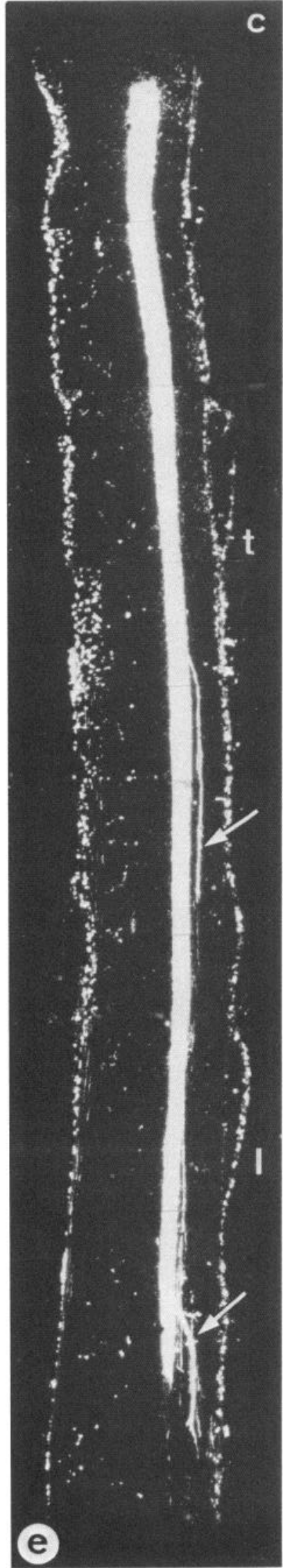

C

level $(f)$ and the presence of CST axons (arrows) within the sensory tracts at the lumbar level $(g) . c$, cervical; $l$, lumbar; $l t$, lower thoracic; $t$, thoracic; $u t$, upper thoracic; $d$, dorsal; $v$, ventral; st, sensory tracts. Magnification: $a-d, 45 \times ; e, 14 \times ; f$ and $g, 42 \times$. 

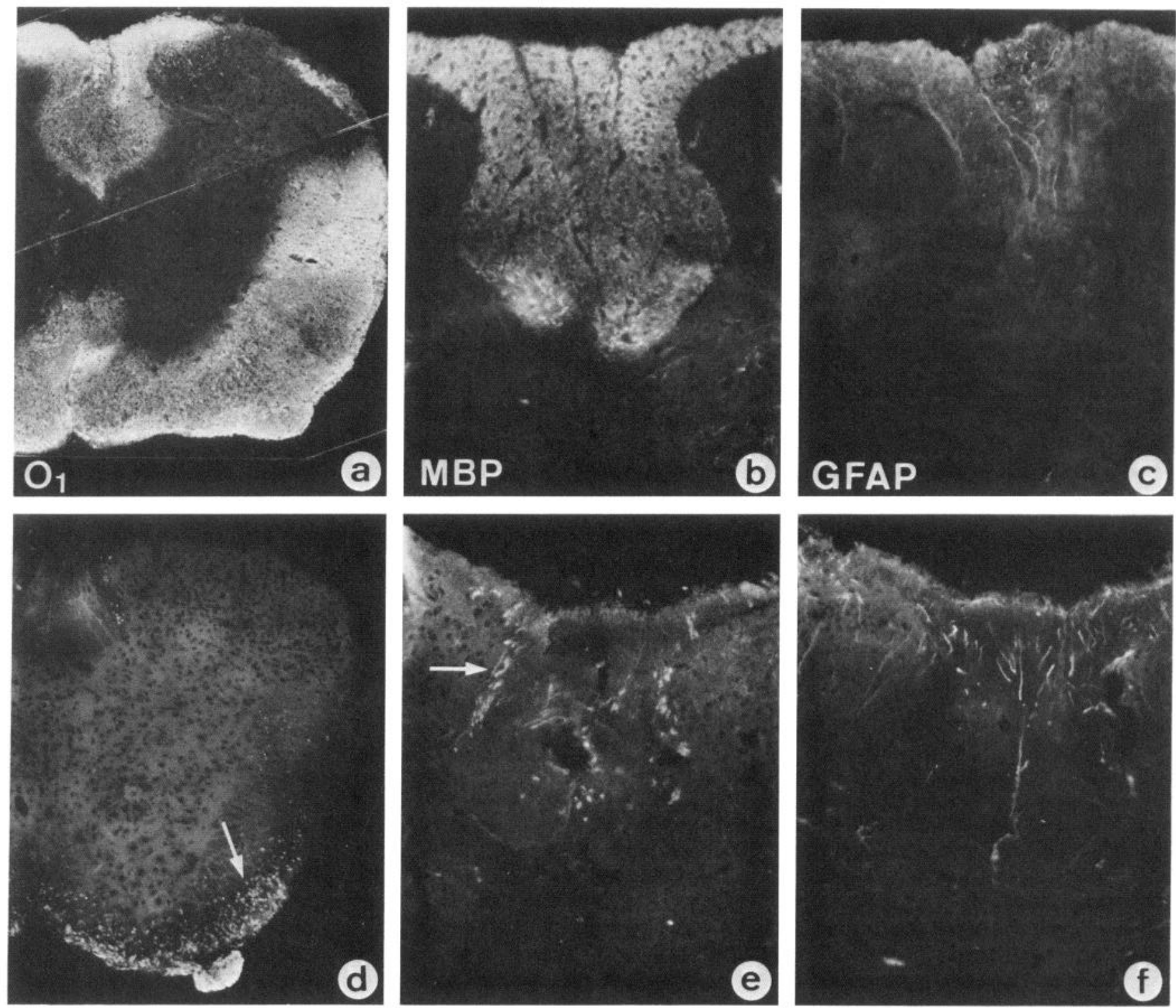

Figure 5. X-irradiation drastically reduces myelin markers GalC (antibody $O_{l}$ ) and $M B P$, but not radial $G F A P$-positive astrocyte processes: thoracic spinal cord of P10 nonirradiated control animals $(a-c)$ and x-irradiated littermates $(d-f)$. $a$ and $d$, Strong GalC fluorescence of dorsal, ventral, and lateral white matter of a normal spinal cord (a) and almost total absence, except for a few axons (arrow), in an x-irradiated spinal cord $(d) . b$ and $e$, Pronounced MBP staining of dorsal columns in controls $(b)$ and virtual absence of MBP in x-irradiated spinal cord $(e)$. Arrow points to remaining myelin around a few axons. $c$ and $f$, Radial GFAP-positive astrocyte processes present in dorsal funiculus of control $(c)$ as well as $\mathrm{x}$ irradiated $(f)$ spinal cord. Magnification: $a$ and $d, 50 \times ; b$ and $d-f, 156 \times$.

of CNS myelin (Caroni and Schwab, 1988b). In order to supply antibodies to the developing spinal cord continuously and in high concentrations, IN-1-producing hybridoma cells were injected (together with a first dose of antibodies) unilaterally in newborn rats into the cortex and lateral ventricle, where they rapidly developed antibody-secreting small tumors (Schnell and Schwab, 1990). High levels of antibodies were present in the CSF and even detectable in peripheral blood (not shown). Myelin formation proceeded normally, as judged from MBP immunostaining at P10 (not shown). All brains were examined at the time of death to ensure that the tumor had not affected the contralateral hemisphere. CST tracing was exclusively done from the intact half of the brain. An anti-HRP antibody-secreting hybridoma line (developed for peroxidase-antiperoxidase reactions) generated from the same parent myeloma line was used as a control. As in myelin-free animals, the cross-sectional areas of the CST showed a significant increase at C5 (by 35\%; $p<$ 0.05 ) and T5 (by $25 \% ; p<0.05$; Fig. 6). The smaller increase of the tract area with IN-1-treated as compared to $\mathrm{x}$-irradiated rats might be due to an incomplete neutralization of the neurite growth inhibitors due to limited antibody penetration. In crosssections, the dorsal aspect of the CST in IN-1-treated rats was more irregular, and fibers and bundles of CST axons entering the sensory tracts were observed (Fig. 9). Such aberrant CST fiber bundles descending in the sensory ascending tracts in the rostral spinal cord became particularly obvious by the analysis of sagittal sections (Fig. 10). As after x-irradiation, outgrowth of CST collaterals into the gray matter and also on the contralateral side of the spinal cord occurred in the IN-1 rats (Fig. 10). The outgrowth of these collaterals shows the striking right-angle configuration, as described for the pontine collaterals of the CST by O'Leary and Terashima (1988). The control antibody- 
treated animals showed no size increase or aberrant fiber growth of the CST.

\section{Discussion}

Oligodendrocytes and CNS myelin of higher vertebrates contain potent, membrane-bound inhibitors of neurite growth (Caroni and Schwab, 1988a,b; Schwab and Caroni, 1988). During CNS development, the time of myelin formation varies in particular regions and fiber tracts, even within the same CNS area (Windle et al., 1934; Bensted et al., 1957; Rozeik and Von Keyserlingk, 1987; Schwab and Schnell, 1989). As shown in the present study, one region where this pattern is particularly obvious is the dorsal funiculus of the rat spinal cord; myelin formation starts early in the ascending scnsory tracts (fasciculus cuncatus) and precedes the ingrowth of the CST axons. At the cervical and upper thoracic level, the myelinating cuneate fibers dorsally surround the ingrowing CST in a channellike manner. This arrangement suggests that the sharp boundary that exists between the CST and the ascending sensory tracts at the cervical and upper thoracic level of the spinal cord could be due to the presence of the myelin-associated neurite growth inhibitors. The finding that CST fascicles do intermix with the ascending sensory tracts at the more caudal levels of the spinal cord also correlates with the more restricted distribution of differentiated oligodendrocytes and myelin constituents at the time when CST fibers grow into the lower thoracic and lumbar segments. Likewise, the "myelin channel" is thin or absent on the ventrolateral and ventral aspect of the CST where the collaterals grow out into the gray matter. These observations suggest a function of negative guidance for myelin-associated neurite growth inhibitors. Thus, in addition to presumable positive cues determining CST position (Joosten and Gribnau, 1989a,b), inhibitor-containing tissue around the CST would exert a "guard-rail" function for the growing axons and would thereby prevent the ingrowth of descending CST fibers into the neighboring ascending sensory tracts. To test this hypothesis, myelin formation was prevented by $\mathrm{x}$-irradiation, or neurite growth inhibitors were neutralized by the specific monoclonal antibody IN-1 (Caroni and Schwab, 1988b).

High-dose $\mathrm{x}$-irradiation administered to newborn rats leads to an almost complete loss of oligodendrocytes and myelin formation in the spinal cord (Gilmore, 1963a,b). The absence of GalC and MBP confirmed the effectiveness of the treatment under our conditions. Radial GFAP-positive processes of astrocytes in the "white matter" appeared undisturbed. Crosssections of irradiated segments showed a doubling of the CST cross-sectional area and the appearance of aberrant CST fibers and fascicles, in particular in the fasciculus cuneatus and gracilis. In normal development, such ectopic CST fibers are totally absent from the ascending sensory tracts in the rostral half of the spinal cord. Some of these aberrant CST fascicles could be followed over long distances in a caudal direction, indicating that they are ectopic CST axons, rather than axon collaterals. The latter observation is also of particular interest, because it points to the absence of rostrally directing cues in the sensory tracts at this age, or alternatively, to the inability of CST fibers to recognize such cues (Dodd and Jessell, 1988).

This conspicuous disturbance of the CST anatomy in the absence of oligodendrocytes is consistent with a channeling function of myelin-containing tissue for the growing CST. However, the irradiation or the absence of oligodendrocytes and
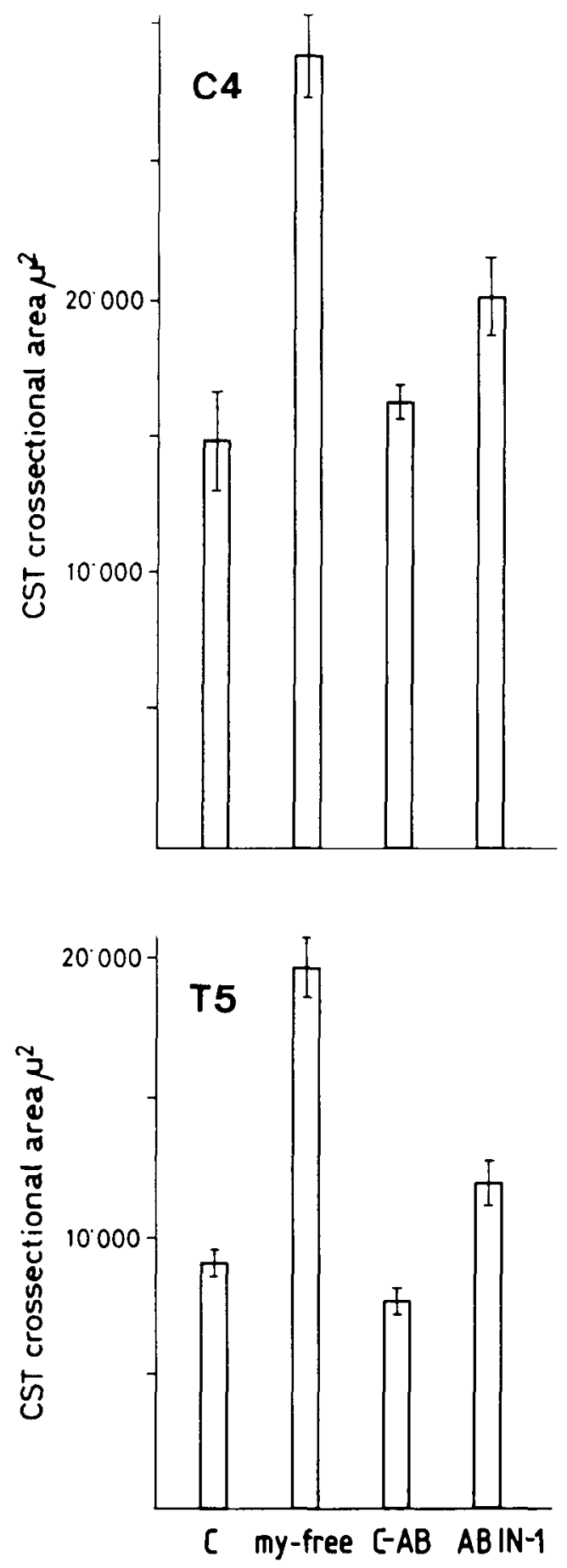

Figure 6. Cross-sectional area of the CST (in $\mu \mathrm{m}^{2}$ ) at the levels $C 4$ and $T 5$ in $P 10$ rats. $C$, untreated control rats. my-free, $\mathrm{x}$-irradiated rats depleted of oligodendrocytes and myelin; $C-A B$, rats with the control anti-HRP antibody; $A B I N-1$, rats treated with antibody IN-1 against neurite growth inhibitors (Caroni and Schwab, 1988b). Mean crosssectional area $\pm \operatorname{SEM}(n=9-17$ for each group) is shown. The difference between antibody IN- 1 and controls in significant at the $p<0.05$ level.

myelin may have led to other effects that could have influenced or induced the aberrant growth of the CST fibers. Although laminin remained restricted to the basement membranes of blood vessels and the spinal cord surface (data not shown), changes in the expression of, for example, other cell adhesion and matrix molecules, could not be excluded. Experiments with IN-1 an- 


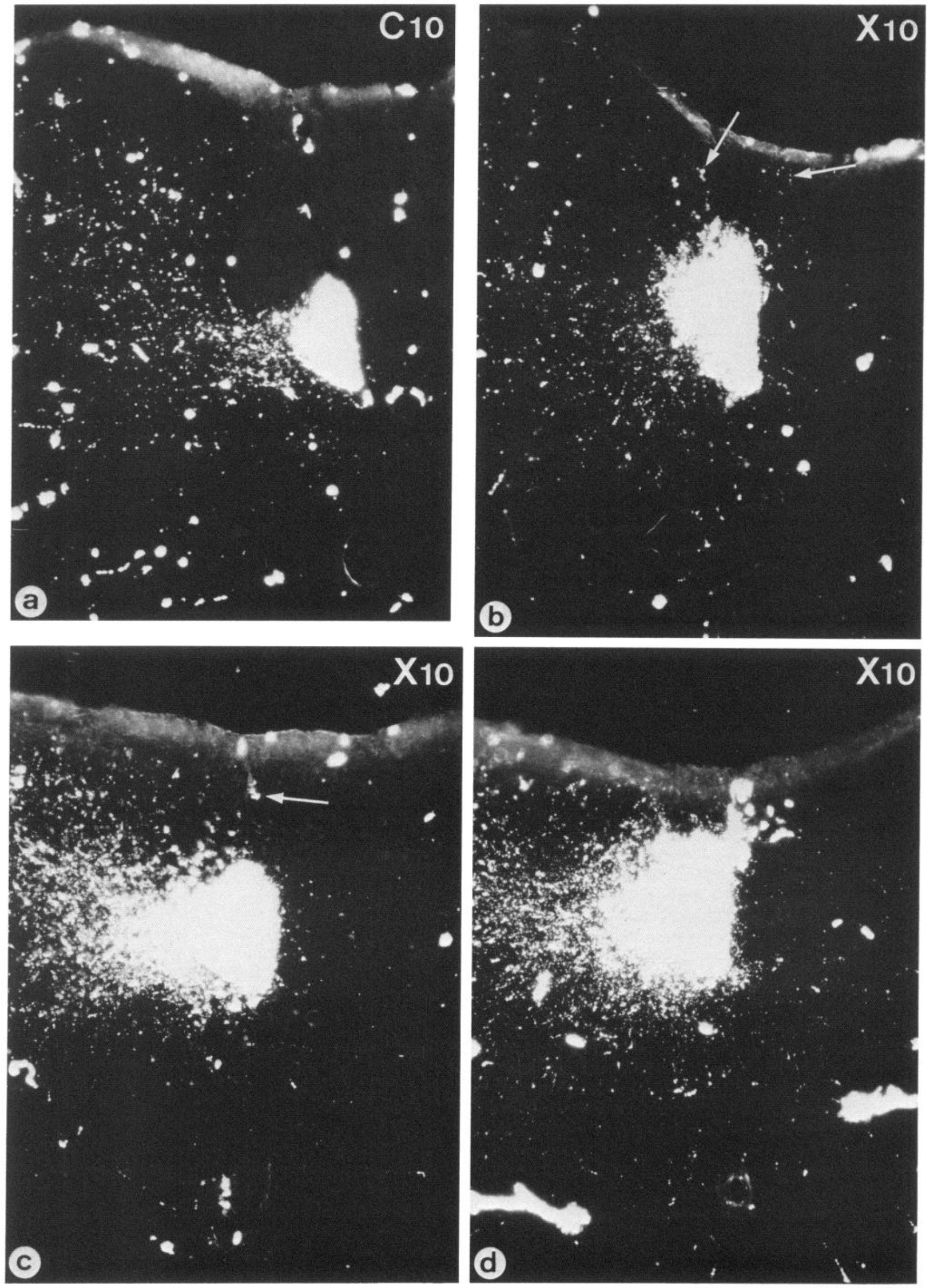



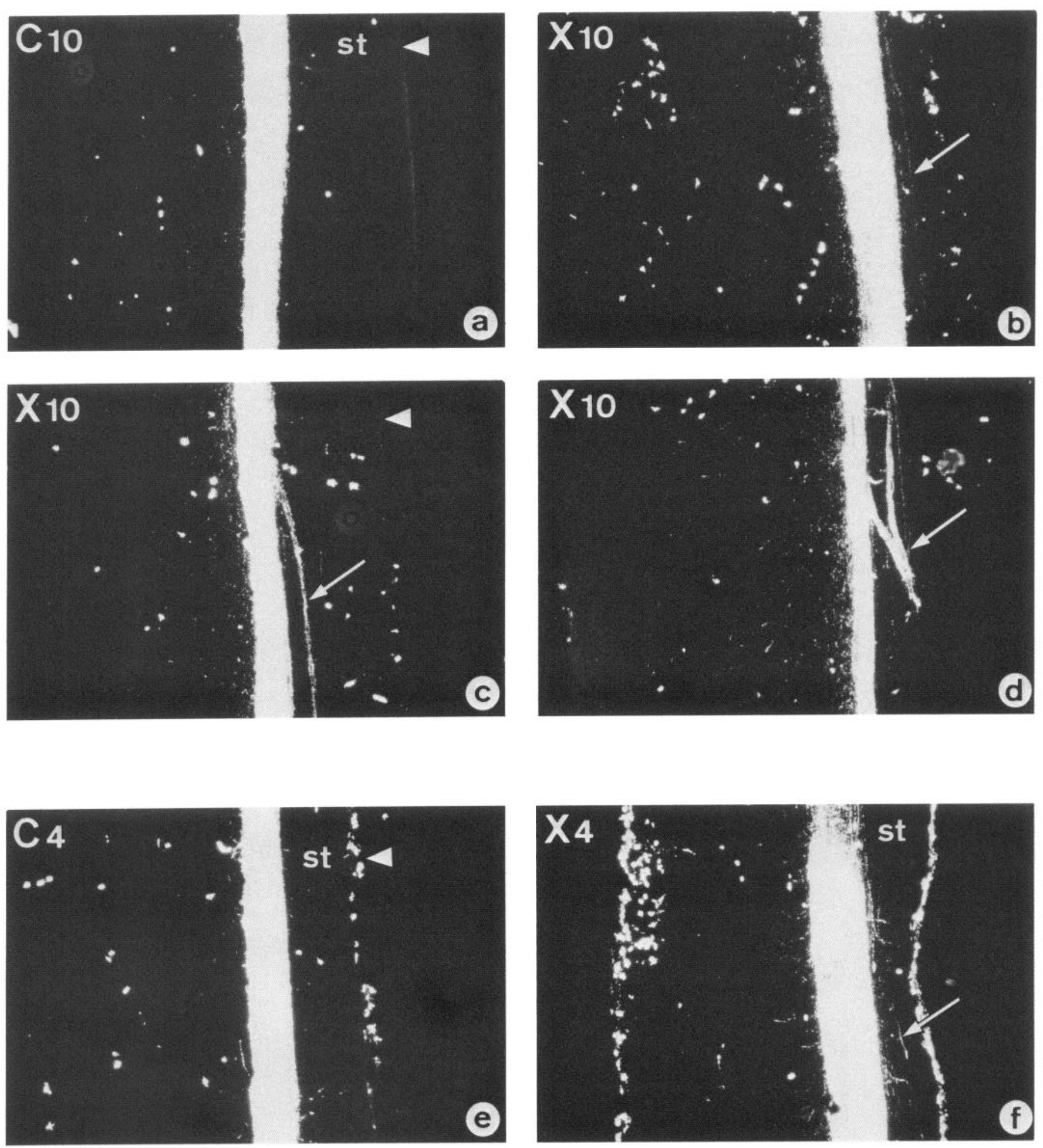

Figure 8. Sagittal sections of spinal cords in $\mathrm{P} 10(a, C 10)$ and $\mathrm{P} 4(e, C 4)$ controls or $\mathrm{P} 10(b-d, X 10)$ and $\mathrm{P} 4(f, X 4) \mathrm{x}$-irradiated rats. Arrows point to aberrant CST fibers within the sensory tracts $(s t)$ of the dorsal funiculi. Note also the increased outgrowth of CST fibers and collaterals at the ventral aspect of the tract. $a, b, e$, and $f$, Upper thoracic; $c$, Middle thoracic; $d$, Lumbar level. Arrow heads, dorsal spinal cord surface. Magnification, $50 \times$.

Figure 7. Cross-sections of dorsal spinal cords at the level T5 with labeled left CST in P10 rats. $a$, Untreated control (C10) $b-d$, X-irradiated, myelin-deficient rats $(X 10)$. Note the size increase of the CST and the scattering of CST fibers in the surrounding tissue, including the ascending sensory tracts (arrows) and the contralateral spinal cord. Magnification, $350 \times$. 

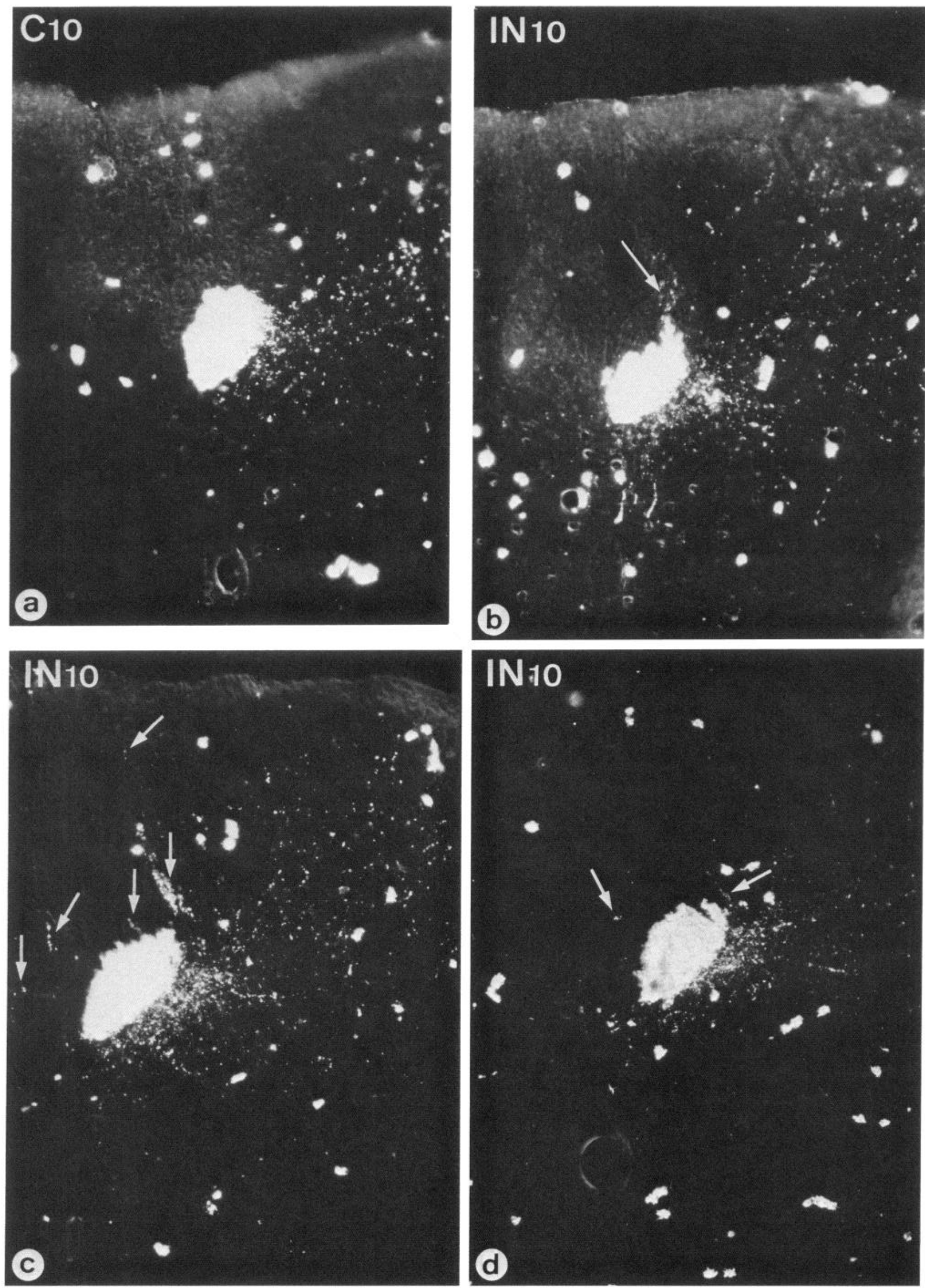

IN 10

2
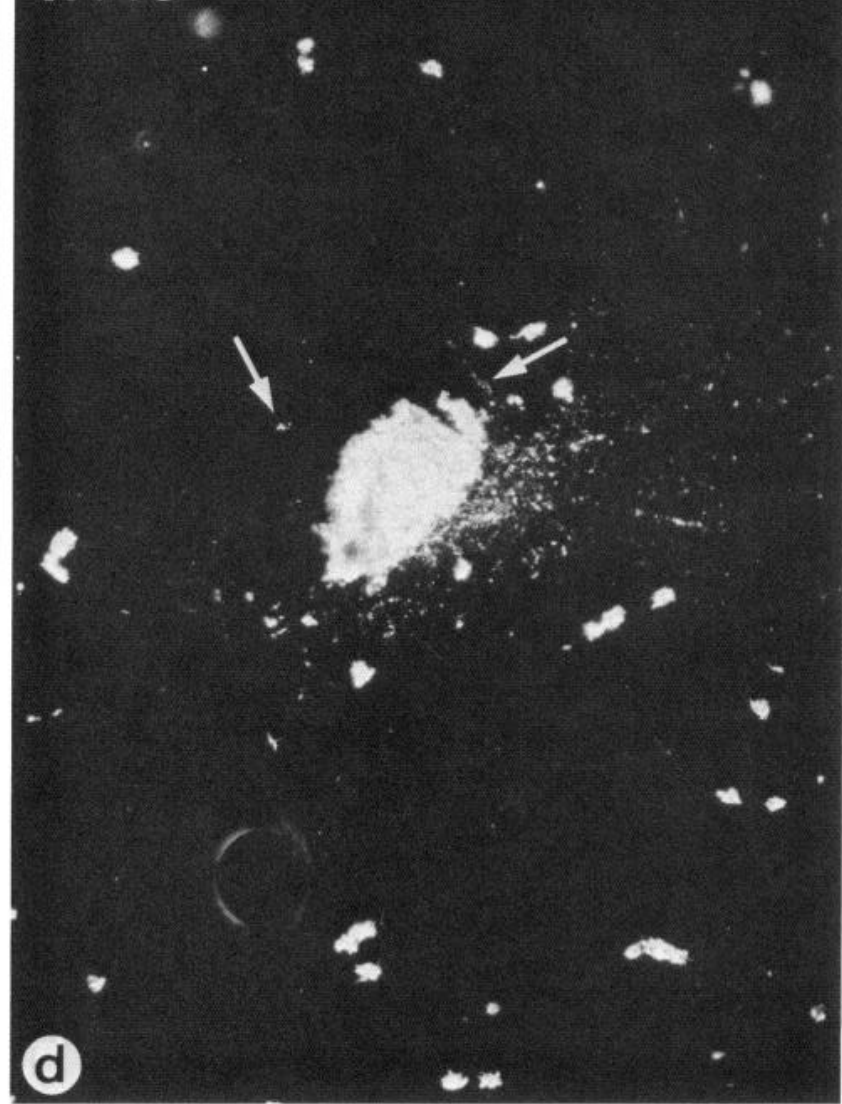

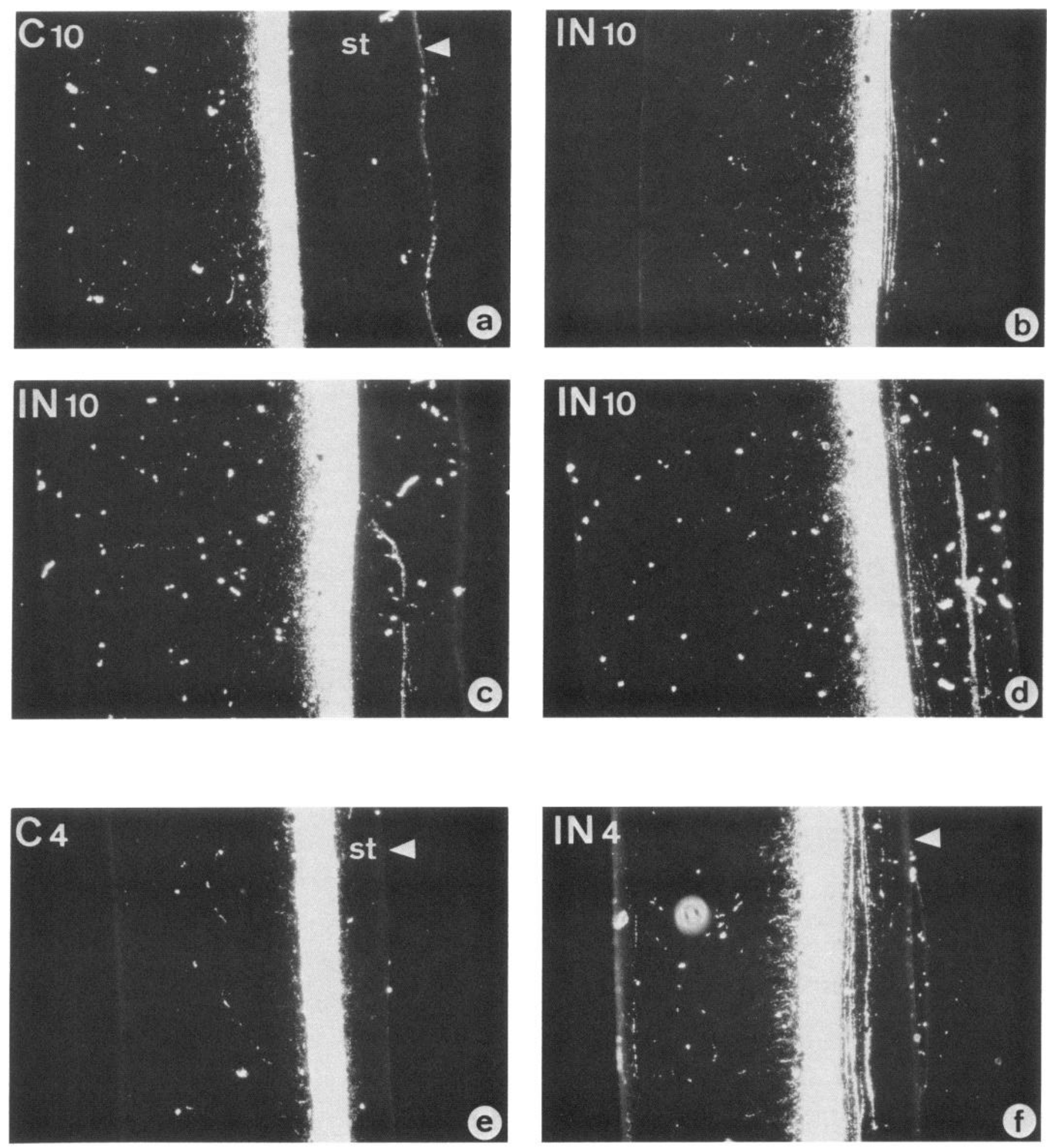

Figure 10. Sagittal sections of middle thoracic spinal cords of antibody-treated rats. $a$, P10 control, anti-HRP-treated rat (C10) showing the welldelineated CST. $b-d$, Many aberrant CST fibers are present in the ascending tracts in IN-1-treated P10 animals (IN10), and increased numbers of collaterals grow out at right angles from the ventral margin of the CST. A picture similar to P10 is seen at P4 for control, anti-HRP-treated (e, $C 4)$, and IN-1-treated $(f, I N 4)$ thoracic spinal cords. st, sensory tracts; arrowheads, dorsal spinal cord surface. Magnification, $50 \times$.

Figure 9. Cross-sections of dorsal spinal cords at T5 showing the labeled right CST in P10 animals. $a$, Control antibody-treated rat (C10) showing sharp delineation of the CST and no CST fibers in the ascending sensory tracts (large dots reflect catalase reaction product in blood vessels). $b-d$, IN-1 antibody-treated rats (IN10). The CST boundary is less regular, and CST fibers/fascicles are found within the sensory tracts (arrows) and on the contralateral side $(b)$. Magnification, $350 \times$. 
tibodies neutralizing the neurite growth inhibitors of CNS myelin were therefore performed.

Implantation of IN-1 antibody-secreting hybridoma cells in 1 hemisphere was a useful way to continuously supply antibodies to the spinal cord via the CSF (Schnell and Schwab, 1990). Although antibody penetration is limited in uninjured adult CNS tissue, extracellular spaces are larger and the junctions and barrier functions are less complete in early postnatal animals, permitting sufficient antibody penetration. Cross-sections of IN- 1 spinal cords at the levels $\mathrm{C} 4$ and $\mathrm{T} 5$ also showed an increase of the CST area. Longitudinal section analysis at P4 and P9-11 showed an increased number of descending CST fibers invading the ascending dorsal sensory tracts, very similar to the situation in $\mathrm{x}$-irradiated rats. Aberrant CST growth was absent in animals with control (anti-HRP) antibody-secreting tumors, thus excluding side effects of the tumor or the immune suppression. Interestingly, the density of collateral growth from the CST into the gray matter was also increased after $\mathrm{x}$-irradiation and by IN-1 treatment. This shows that the scattered oligodendrocytes present on the ventrolateral border of the CST also exert inhibitory effects that restrain collateral outgrowth.

Several conclusions can be drawn from the present experiments: (1) Oligodendrocytes, and in particular, their membrane components, the neurite growth inhibitors NI-35 and NI-250, can restrict the access of late-growing fibers to myelinating CNS territories. For the CST in the rostral half of the spinal cord, they exert a channeling and "guard-rail" function to keep the CST axons in a compact tract and prevent the ingrowth into the neighboring sensory tracts. (2) Although many ectopic fibers occurred and the CST area was enlarged, a majority of the CST fibcrs were still growing in the appropriate anatomical region. This points to the presence of positive guidance cues acting on the growing CST axons (Joosten and Gribnau, 1989a,b). (3) The observation of ingrowth and long-distance elongation of descending CST fibers in the ascending sensory tracts indicates that CST axons possess their own directionality irrespective of the direction and modality of the tracts in which they grow.

The role of the myelin-associated neurite growth inhibitors in the adult CNS is still unclear. Their absence or neutralization led to long-distance fiber regeneration from the transected CST (Savio and Schwab, 1990; Schnell and Schwab, 1990). A possible role of these inhibitors for the stabilization of fiber tracts and the local restriction of sprouting remains to be investigated.

\section{References}

Bandtlow CE, Zachleder T, Schwab ME (1990) Oligodendrocytes arrest neurite growth by contact inhibition. J Neurosci 10:3837-3848.

Bensted JPM, Dobbing J, Morgan RS, Reid RWT, Payling Wright G (1957) Neuroglial development and myelination in the spinal cord of the chick embryo. J Embryol Exp Morphol 5:428-437.

Caroni P, Schwab ME (1988a) Two membrane protein fractions from rat central myelin with inhibitory properties for neurite growth and fibroblast spreading. J Cell Biol 106:1281-1288.

Caroni P, Schwab ME (1988b) Antibody against myelin-associated inhibitors of neurite growth neutralizes nonpermissive substrate properties of CNS white matter. Neuron 1:85-96.

Caroni P, Schwab ME (1989) Codistribution of neurite growth inhibitors and oligodendrocytes in rat CNS: appearance follows nerve fiber growth and precedes myelination. Dev Biol 136:287-295.

Chiquet-Ehrismann R, Kalla P, Pearson CA, Beck K, Chiquet M (1988) Tenascin interferes with fibronectin action. Cell 53:383-390.

Dodd J, Jessell TM (1988) Axon guidance and the patterning of neuronal projections in vertebrates. Science 242:692-699.

Gilmore SA (1963a) The effects of $x$-irradiation on the spinal cords of neonatal rats. I. Neurological observations. I Neuropathol Exp Neurol 22:285-293.

Gilmore SA (1963b) The effects of $x$-irradiation on the spinal cords of neonatal rats. II. Histological observations. J Neuropathol Exp Neurol 22:294-301.

Gribnau AAM, De Kort EJM, Dederen PJWC, Nieuwenhuys R (1986) On the development of the pyramidal tract in the rat. Anat Embryol 175:101-110.

Hankin MH, Silver J (1988) Development of intersecting CNS fiber tracts: the corpus callosum and its perforating fiber pathway. J Comp Neurol 272:177-190.

Jessell TM (1988) Adhesion molecules and the hierarchy of neural development. Neuron 1:3-13.

Joosten EAJ, Gribnau AAM (1989a) Astrocytes and guidance of outgrowing corticospinal tracts axons in the rat. An immunocytochemical study using anti-vimentin and anti-glial fibrillary acidic protein. Neuroscience 31:439-452.

Joosten EAJ, Gribnau AAM (1989b) Immunocytochemical localization of cell adhesion molecule $L 1$ in developing rat pyramidal tract. Neurosci Lett 100:94-98.

Kapfhammer JP, Raper JA (1987) Interactions between growth cones and neurites growing from different neural tissues in culture. $J$ Neurosci 7:1595-1600.

Kapfhammer JP, Gruenewald BE, Raper JA (1986) The selective inhibition of growth cone extension by specific neurites in culture. $\mathrm{J}$ Neurosci 6:2527-2534.

Matthews MA, Duncan D (1971) A quantitative study of morphological changes accompanying the initiation and progress of myelin production in the dorsal funiculus of the rat spinal cord. J Comp Neurol 142:1-22.

Mesulam M-M (1978) Tetramethyl benzidine for horseradish peroxidase neurohistochemistry: a non-carcinogenic blue reaction-product with superior sensitivity for visualizing neural afferents and efferents. J Histochem Cytochem 26:106-117.

O'Leary DDM, Terashima T (1988) Cortical axons branch to multiple subcortical targets by interstitial axon budding: implications for target recognition and "waiting periods." Neuron 1:901-910.

Raper JA, Kapfhammer JP (1990) The enrichment of a neuronal growth cone collapsing activity from embryonic chick brain. Neuron 2:21-29.

Rozeik C, Von Keyserlingk D (1987) The sequence of myelination in the brainstem of the rat monitored by myelin basic protein immunohistochemistry. Dev Brain Res 35:183-190.

Sanes JR (1983) Roles of extracellular matrix in neural development. Annu Rev Physiol 45:581-600.

Savio T, Schwab ME (1990) Lesioned corticospinal tract axons regenerate in myelin-free rat spinal cord. Proc Natl Acad Sci USA 87: 4130-4133.

Schnell L, Schwab ME (1990) Axonal regeneration in the rat spinal cord produced by an antibody against myelin-associated neurite growth inhibitors. Nature 343:269-272.

Schreyer DJ, Jones EG (1982) Growth and target finding by axons of the corticospinal tract in prenatal and postnatal rats. Neuroscience 7 : $1837-1853$.

Schreyer DJ, Jones EG (1988) Axon elimination in the developing corticospinal tract of the rat. Dev Brain Res 38:103-1 19.

Schwab ME, Caroni $P$ (1988) Oligodendrocytes and CNS myelin are non-permissive substrates for neurite growth and fibroblast spreading in vitro. J Neurosci 8:2381-2393.

Schwab ME, Schnell L (1989) Region-specific appearance of myelin constituents in the developing rat spinal cord. J Neurocytol 18:161169.

Silver J, Poston M, Rutishauser U (1987) Axon pathway boundaries in the developing brain. I. Cellular and molecular determinants that separate the optic and olfactory projections. J Neurosci 7:2264-2272.

Sommer I, Schachner M (1981) Monoclonal antibodies $\left(\mathrm{O}_{1}\right.$ to $\left.\mathrm{O}_{4}\right)$ to oligodendrocyte cell surfaces: an immunocytological study in the central nervous system. Dev Biol 83:311-327.

Steindler DA, Cooper NGF (1987) Glial and glycoconjugate boundaries during postnatal development of the central nervous system. Dev Brain Res 36:27-38.

Steindler DA, Cooper NGF, Faissner A, Schachner M (1989) Boundaries defined by adhesion molecules during development of the cerebral cortex: the $\mathrm{J} 1 /$ tenascin glycoprotein in the mouse somatosensory cortical barrel field. Dev Biol 131:243-260. 
Vielmetter J, Stürmer CAO (1989) Goldfish retinal axons respond to position-specific properties of tectal cell membranes in vitro. Neuron 2:1331-1339.

Walter J, Henke-Fahle S, Bonhoeffer F (1987) Avoidance of posterior tectal membranes by temporal retinal axons. Development 101:909913.
Windle WF, Fisch MW, O'Donnell JE (1934) Myelogeny of the cat as related to development of fiber tracts and prenatal behavior patterns. J Comp Neurol 59:139-165. 